\title{
Toward a Wide Angle Tomographic Atom Probe
}

\author{
L. Renaud
}

CAMECA, 103 Bd. St-Denis, 92403, Courbevoie cedex, France.

Three dimensional Atom Probe (3DAP) technique can be considered as the only technique witch allows 3D imaging capabilities at the atomic scale and measurement compositions [1] (Figure 1). Since its early developments, 3D Atom probe has demonstrated his major contribution in materials science [2]. However, today most of the 3D AP instruments suffer from a small field of view (typically $15 \mathrm{~nm} \times 15 \mathrm{~nm}$ ) so that the amount of material imaged from a single analysis makes it difficult to detect small objects of interest if their concentrations are too small.

To overcome this limitation, CAMECA in collaboration with the GPM at Rouen University (France) is developing a new 3D atom probe : the Wide Angle Tomographic Atom Probe (WATAP). This new instrument will allow a large field of view ( 25 bigger than a conventional 3D AP).

The increase of the field of view can be easily achieved by positioning the PSD (position sensitive detector) close to the specimen (the field of view is proportional to the inverse of the distance between the specimen and the detector). However, reducing the flight path length increases the probability to have multi-hits events (events on which more than one ion arrive on the detector see figure 2). Therefore, a wide-angle configuration requires the use of a PSD with high performances in term of timing accuracy and multi-hits capabilities, otherwise quantitative analysis cannot be achieved. Indeed, the precision of the concentration measurements depends directly of the rate of loss of the detection system : each single atom must detected .

To design the WATAP, a new position sensitive detector has been specially developed by Rouen's group. This new detector has higher performances than the existing detections systems used in 3D atom probe techniques. It allows the detection of several ions evaporated on the same pulse. These ions can strike the detector at the same time or with times of flight different from a few nanoseconds. For each detected ion, position and time of flight are accurately determined. This detector is based on a standard two-dimensional delay line [3] coupled to a fast analog digitalizer electronic system (Figure 3). The performances of this new detection system allow fast acquisition frequencies with a timing accuracy below $150 \mathrm{ps}$ and a dead time less than $1.5 \mathrm{~ns}$.

The goal of this presentation will be to introduce of this new instrument that allows a large field of view while preserving quantitative measurements. Some preliminary results obtained with the prototype will be presented in order to evaluate the performances of this instrument. In the last part of the presentation, new developments and future applications of the instrument will be discussed.

\section{References}

[1] D. Blavette et al., Nature 363 (1993) 432 - 434

[2] D. Blavette et al., Science Dec 17 (1999) 2317-2319

[3] O. Jagutzki et al., Nucl. Instr. and Meth. in Phys. Res. A, 477 (2002) 244-249 


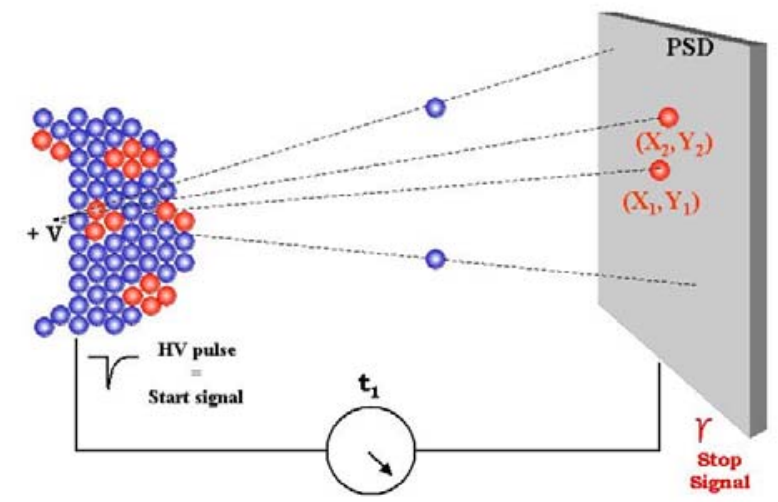

Figure 1 : Principle of the 3D atom probe technique.

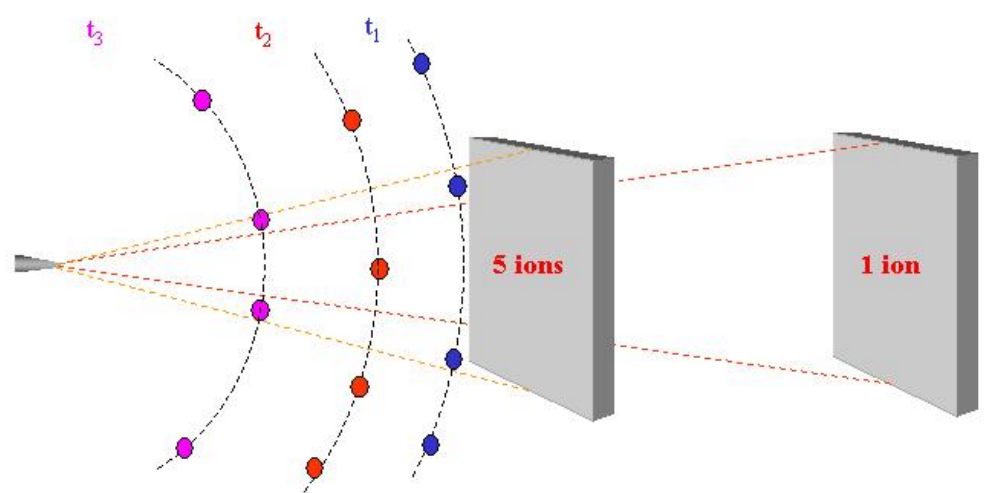

Figure 2 : Increasing field of view involves increasing multi-hits capabilities of the detector.

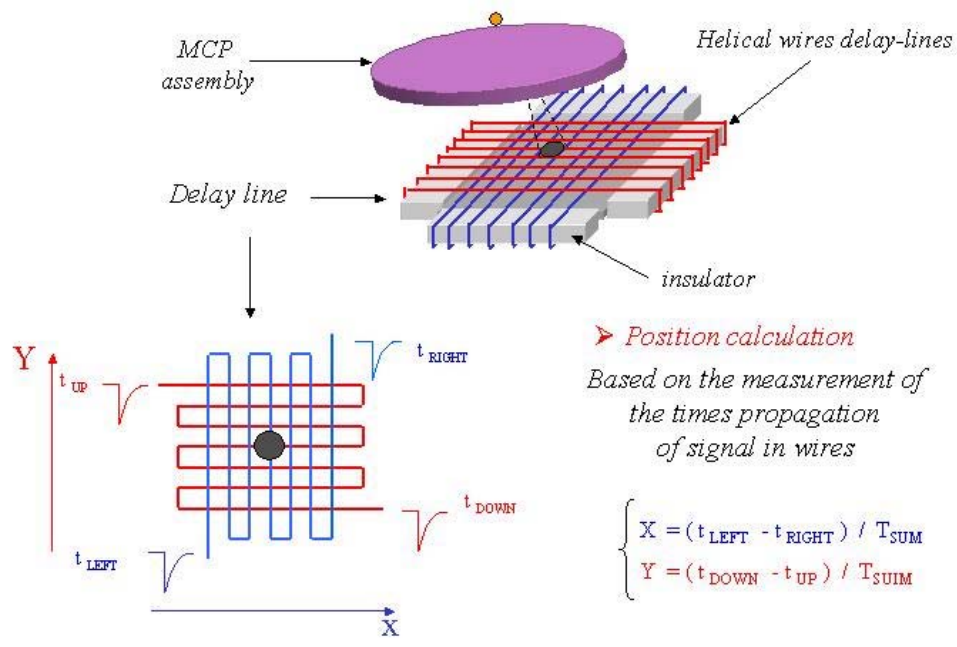

Figure 3 : Principle and design of the new position sensitive detector . 